\title{
LARGE-SCALE CLUSTERING OF GALAXIES WITH MASSIVE DARK HALOS. II. MASS-TO-LIGHT PROFILES AND DYNAMICAL MASS ESTIMATES OF GALAXY GROUPS
}

\author{
August E. Evrard \\ Physics Department, SUNY-Stony Brook, and Institute of Astronomy, University of Cambridge \\ Received 1986 September 8 ; accepted 1986 October 22
}

\begin{abstract}
Properties of groups of galaxies evolved within large-scale clustering simulations are examined. Models begun with 800 galaxies imbedded in extended dark-matter halos were run in flat $(\Omega=1)$ and open $\left(\Omega_{f}=\right.$ $0.15)$ cosmologies. Each galaxy is represented by a single "luminous" particle and its halo comprised of 25 "dark" particles. Ninety percent of the mass is in the dark component. For comparison, similar runs with no dark matter were performed for each cosmology.

A group-finding algorithm is applied to the final model configurations with the aim of identifying wellrelaxed groups in virial equilibrium. Comparison of the four model catalogs with an observational catalog compiled by Huchra and Geller shows good agreement in the frequency of small groups, but observed group sizes and velocity dispersions are not reproduced within any single model.

Mean mass and "light" profiles exhibit evidence for segregation of galaxies from dark matter. The integrated mass-to-light ratio, $M / L(<r)$, defined as the ratio of total to luminous mass measured outward from the group centroid, rises as roughly $r^{0.5}$ from values of $\sim 2$ in the central regions to a value $M / L(<r) \approx 6$, thereafter rising more slowly to the global mean value $M / L_{\text {glob }}=10$ at distances of $\sim 6 \mathrm{Mpc}(\Omega=1)$ and $\sim 2$ Mpc $\left(\Omega_{f}=0.15\right)$. This mean behavior is independent of the number of galaxies in the group.

In the models without dark matter, median virial mass estimates for groups with three or more members are accurate to within $20 \%$. In the dark-halo models, median virial masses derived from galaxy information alone systematically underestimate total group masses by large factors. In terms of mass-to-light ratio, the median virial $M / L$ underestimates the global value by a factor of at least 3 . Estimates of $\Omega_{0}$ based on observed group $M / L$ ratios should be subject to this systematic error if the total mass in real groups is dominated by an extended dark component. The value $\Omega_{0} \approx 0.2$ determined this way from observations could thus be reconciled with a true value of $\Omega_{0}=1$.

The models show that dynamical segregation of galaxies from dark matter is capable of "biasing" the galaxy distribution such that it ends up tracing $\sim 35 \%$ of the total mass in the universe. The rest of the mass lies in extended halos around individual galaxies, binaries, small groups, and clusters. This dynamical biasing occurs without any initial large-scale bias in the galaxy distribution, since galaxies in the models initially trace all the mass on scales comparable to the mean intergalactic separation.
\end{abstract}

Subject headings: cosmology — galaxies: clustering — galaxies: structure - numerical methods

\section{INTRODUCTION}

Virial mass estimates of galaxy groups provided some of the seminal evidence for the existence of dark matter in the universe (Zwicky 1933; Smith 1936). Mass-to-light ratios in the range $100-250 h_{50}$ (where $H_{0}=50 h_{50} \mathrm{~km} \mathrm{~s}^{-1} \mathrm{Mpc}^{-1}$ ) are consistently measured (Geller and Peebles 1973; Turner and Gott 1976; Rood and Dickel 1978; Huchra and Geller 1982; see also Faber and Gallagher 1979). Although one order of magnitude more than galactic $M / L$ 's, these values are still much less than that required to provide closure density

$$
(M / L)_{c}=3 H_{0}^{2} / 8 \pi G \rho_{l} \approx 700 h_{50},
$$

given the mean luminosity density $\rho_{l}=10^{8} L_{\odot} \mathrm{Mpc}^{-3}$ (Felten 1985). Simple estimates of $\Omega_{0}$ have been inferred from median group mass-to-light ratios by Gott and Turner (1976) and Geller (1982) by taking the ratio

$$
\Omega_{0}=(M / L) /(M / L)_{c} \approx 0.2 .
$$

If galaxies trace all the mass on scales of a few Mpc or less (typical group sizes), then the estimate above reflects the true mass density in the universe, provided the galaxies in groups satisfy the virial condition (i.e., the groups are "virialized"). Simple top-hat arguments (see Rees and Ostriker 1977) argue that virialization should occur at overdensities $\delta \rho / \rho \gtrsim 200$. The galaxy overdensities in observed groups are of this order, and the group crossing times are short, so the galaxies are presumed in virial equilibrium. Numerical experiments (Turner et al. 1979; Heisler, Tremaine, and Bahcall 1985; Evrard and Yahil $1985 a, b$ ) have verified the fact that virial equilibrium is established in such clustered regions and that mass estimates can be made accurately, to within a factor of 2 or better. These $N$-body simulations assumed all mass was contained in galaxies and treated galaxies as softened point particles interacting via elastic gravitational encounters.

The situation may be quite different if galaxies are not fair tracers of the total mass distribution on clustered scales. The first paper in this series (Evrard 1986, hereafter Paper I) introduced numerical simulations of large-scale clustering of galaxies formed within massive dark halos. The initial distribution consisted of 800 randomly placed galaxies, each represented by a single "luminous" particle, and each surrounded by an 
isothermal-profile halo of 25 " dark" particles. The halos contained $90 \%$ of the total mass and were truncated at radii comparable to the initial intergalactic spacing $d_{i}$, implying galaxies initially traced the mass on scales $r \gtrsim d_{i}$. In Paper I, it was found that the two-point galaxy correlation function $\xi(r)$ was enhanced on small scales relative to similar models with no dark matter. Visual inspection of clustered regions showed that the galaxies lay preferentially nearer the centers of local density enhancements. It was clear that, as the galaxies hierarchically clustered, segregation of the galaxies from the dark matter had occurred.

In this paper, the degree of segregation is investigated by measuring the mass and light profiles of galaxy groups in the final configurations of the models. The ability of the virial theorem to measure the amount of mass around galaxies, as well as the mean cosmological mass density, is tested. A "friends-of-friends" algorithm is used to identify the groups. To assess how realistic are the simulations, properties of the groups in the simulated catalogs are compared to data from an observed catalog of nearby groups constructed by Huchra and Geller (1982, hereafter HG).

For each group, the amount of total and luminous mass is measured as a function of distance from the centroid of the group's galaxies. Mean profiles are established for ensembles of groups with given memberships $N_{g}$. For isolated galaxies $\left(N_{g}=1\right)$, expected rotation curves are estimated from knowledge of $M_{\text {tot }}(r)$. For all groups, mass-to-light profiles, $M / L(<r)$, are defined by the ratio of total to luminous mass within $r$ of the centroid. The ratio is found to increase with $r$ in a manner independent of $N_{g}$. A measure of the "local" $M / L$ for each group is taken as the mass-to-light ratio within $r_{*}$ of the centroid, where $r_{*}$ is the distance to the farthest galaxy in the group. The median value of $\sim 3.5$ for this quantity indicates that only $\sim 35 \%$ of the total mass initially associated with the galaxies actually ends up within the volume of the group occupied by the clustered galaxies at the final epoch.

Standard virial mass estimates are made for all groups in the catalogs. Several variants of the standard form, detailed in $\S \mathrm{V}$, are also considered. Resultant mass-to-light ratios are defined by the ratio of virial estimated mass to known luminous mass in the group. Comparison of these values to the local $M / L$ 's defined above and the global mean value indicates that the virial theorem can accurately measure the amount of mass associated locally with the galaxies $\left(M / L\right.$ within $\left.r_{*}\right)$, but the value of $\Omega_{0}$ is seriously underestimated because of the segregation which has taken place in groups and clusters. These conclusions confirm those of Barnes (1984), who simulated the clustering of individual groups dominated by dark matter.

A brief description of the models, including initial conditions, is given in $\S \mathrm{II}$. The subsequent section describes the group finding algorithm and presents the model catalogs produced by its application. Group properties are compared to the observational catalog of $\mathrm{HG}$. In $\S \mathrm{IV}$, the group mass and light profiles are presented along with the median local $M / L$ values. Details of the virial analysis are given in $\S \mathrm{V}$, and results presented and discussed. A final section attempts to summarize and interpret the main results.

\section{THE MODELS}

The final configurations of the four simulations used in Paper I are analyzed. Two models each for a flat and open universe were simulated, one with dark-matter halos around galaxies (labeled DM), the other with all mass in single-particle galaxies (labeled SP). The $\Omega=1.0$ models were expanded by a factor of $R=5.4$, while the $\Omega_{f}=0.15$ models were expanded by $R=11.7$, these factors coming from a simple theoretical estimate rather than matching the two-point correlation function, $\xi(r)$. The final configurations are scaled to physical units by assuming a Hubble constant of $50 \mathrm{~km} \mathrm{~s}^{-1} \mathrm{Mpc}^{-1}$ and using the number density of observed bright galaxies to provide a length scale. This yields a length of $63 \mathrm{Mpc}$ for the simulated periodic cube and a mass of $2.2 \times 10^{13} \Omega_{f} M_{\odot}$ for each galactic system.

The initial density distribution of the galaxies' centers of mass is Poissonian. The same random realization of galaxies' positions is used in all four runs. Galactic peculiar velocities $\boldsymbol{v}_{p}$ are assigned at the start by measuring the local peculiar acceleration $g_{p}$ and using the linear theory relationship $v_{p}=$ $\frac{2}{3} H_{0}^{-1} \Omega^{-0.4} g_{p}$.

In the DM models, 25 "dark" particles are assigned around each galaxy, which is represented by a single "luminous" particle. Ninety percent of the total mass is assumed to be dark. Thus, the global "mass-to-light" ratio is 10 in these runs. The density distribution of dark particles within the halos is initially isothermal, following

$$
\rho(r)=\frac{v_{\mathrm{rot}}^{2}}{4 \pi G r^{2}},
$$

truncated at a radius $r_{t}$. The truncation radius is dictated by $\Omega$, the total amount of expansion $R_{f}$, and by constraining the value of the rotation velocity, which is assumed to be $v_{\text {rot }}=200$ $\mathrm{km} \mathrm{s}^{-1}$. This leads to values of $r_{t}=2.1 \Omega_{f} \mathrm{Mpc}$. In terms of the initial mean intergalactic spacing, $d_{i}=L_{i} / N_{\mathrm{gal}}^{1 / 3}$, this is

$$
\frac{r_{t}}{d_{i}}= \begin{cases}1.6, & \Omega=1.1, \\ 0.6, & \Omega_{f}=0.15\end{cases}
$$

Halos in the low-density model are initially distinct, while those in the flat model overlap to create a much smoother dark-matter background among the galaxies.

The velocity field of the dark matter is assigned in similar fashion to the galactic centers of mass, but since $g_{p}$ formally diverges in the centers of the halos, a nonlinear correction to $\boldsymbol{v}_{p}$ is applied and a threshold velocity is imposed. This prevents the occurrence of large peculiar velocities in the centers of the halos while retaining the infall behavior in the outer regions.

The initial phase space configuration of each SP model is identical to that of the luminous population in the corresponding DM run. The mass of each galaxy is now 10 times the mass of a galaxy in the latter, and the global mass-to-light ratio is now unity, since all mass is in galaxies.

The $P^{3} M N$-body program, available courtesty of Dr. G. Efstathiou and his collaborators, is used to integrate the models. The two-body force is softened between particle pairs through use of the potential $\Phi(r)=-G m^{2} /\left(r^{2}+\epsilon^{2}\right)^{1 / 2}$. The softening length $\epsilon$ is held constant in the proper frame and has values of $27 \mathrm{kpc}\left(\Omega_{f}=0.15\right)$ and $58 \mathrm{kpc}(\Omega=1.0)$. The same softening is used for both luminous-luminous and luminousdark interactions. The DM models took $\sim 100 \mathrm{hr}$ of CPU each on a VAX 11/780, while each SP run took $\sim 2 \mathrm{hr}$. More details concerning the initial models can be found in Paper I.

\section{GROUP SAMPLE}

\section{a) Algorithm}

The "friends-of-friends" group finding algorithm has been employed in work with both simulated and real galaxy samples 
(Efstathiou, Fall, and Hogan 1979; HG; Efstathiou and Barnes 1983; Davis et al. 1985). Given a critical distance $r_{c}$, one searches for neighbors of each particle lying within $r_{c}$-its "friends." Starting with any particle, that particle and its friends are labeled as a group. One then runs over the friends in the group and adds their friends, repeating until no new friends are found. This process is repeated until all particles have been assigned to groups.

The friends-of-friends algorithm is attractive for several reasons. It produces a unique catalogue for a given $r_{c}$. All particles are assigned to groups (groups of one member are allowed), and no particle is assigned to more than one group. Asymmetry in groups' mass distributions is not destroyed. Also, the catalogs produced are nested. Given $r_{1}<r_{2}$, all groups found with cutoff $r_{c}=r_{1}$ will be in the catalog with $r_{c}=r_{2}$.

Only one parameter, $r_{c}$, determines the properties of the group catalog. The critical distance used here is $r_{c}=1 \mathrm{Mpc} \approx$ $0.15 d_{f}$, where $d_{f}$ is the mean intergalactic spacing at the final epoch. This value was chosen with the aim of selecting out the high-density $\left(\delta \rho / \rho \gtrsim\left(d_{f} / r_{c}\right)^{3} \approx 300\right)$, presumably virialized, regions in the models. Groups in catalogs produced using $r_{c}=$ $2 \mathrm{Mpc}$ were visibly more subclustered than those in the $r_{c}=1$ Mpc catalog. Often, two distinct groups of three or four members in the latter were identified as a single group with the larger cutoff. Subclustering will generally bias dynamical mass estimates toward larger values, by increasing distances while velocities remains roughly constant $\left(M_{\mathrm{dyn}} \propto r v^{2}\right)$. This effect will be even larger in redshift space, where velocity dispersions will also be artificially increased, as in the case of the Cancer cluster (Bothun et al. 1983). To avoid this contamination, $r_{c}=$ $1 \mathrm{Mpc}$ is adopted. The results of the virial analysis performed in $\S \mathrm{V}$ verify this as a good choice for selecting clustered regions in virial equilibrium.

\section{b) Group Multiplicity Functions}

Figure 1 shows the multiplicity functions of the group catalogs for each of the four runs. Groups with $N_{g} \leq 3$ are shown in the plot, and counts of binaries and singles are tabulated in the inset. The differences among the runs in this statistic are slight. This is not too surprising in view of the fact that the runs began with identical galaxy positions. The largest groups found in the DM models have fewer members than their SP counterparts, as expected from considerations of the reduced peculiar accelerations on scales of a few Mpc outlined in Paper I. The $\Omega=1.0 \mathrm{DM}$ run shows an excess of singles due to the same effect, enhanced in this model by the fact that the initial halo radius is nearly twice the intergalactic separation.

The largest group is found in the $\Omega=1.0 \mathrm{SP}$ model and contains 34 galaxies. The largest in the $\Omega_{f}=0.15 \mathrm{DM}$ run contains only 22 galaxies. Clearly, these simulations are not designed to reproduce the richest clusters observed in nature, which contain hundreds of galaxies. However, such objects are sufficiently rare that they contain only a small fraction of all galaxies (Kaiser and Davis 1985). The majority of galaxies reside in small groups and binaries. It is just these types of systems which populate the models presented here.

A study of nearby groups using the friends-of-friends algorithm in redshift space was done by HG. The critical distance criterion was a combination of projected distance $r_{p}<1.26 h_{50}^{-1}$ Mpc and velocity difference $v_{i j}<400 \mathrm{~km} \mathrm{~s}^{-1}$. A total of 92 groups with $N_{g} \geq 3$ were found among the 1208 galaxies in the sample. Two rich clusters, Virgo, with 166 galaxies, and Ursa Major-Coma, containing 90 galaxies, were among the groups identified. The next largest group after these two contained only 30 galaxies.

Table 1 lists the number and fraction (in parentheses) of galaxies in groups of different membership for the HG catalog and the four simulations. Data for the observed catalog are presented with and without the two rich clusters included. These two observed data sets bracket fairly closely the values found in the simulations. With the rich clusters included, the observed fraction of galaxies in groups with $N_{g}>4$ is increased substantially, and the models contain no such large associations to match this figure. On the other hand, the fractions of galaxies in binaries, triplets and quartets, which vary only a few percent with or without the inclusion of Virgo and Coma, are remarkably similar to the fractions found in the models.

\section{c) Group Sizes and Velocity Dispersions}

More sensitive indicators of dynamics than the multiplicity function are properties such as group size and velocity dispersion. Figures 2 and 3 exhibit scatter diagrams and histograms of these two quantities in each of the four runs. Only groups with $N_{g} \geq 3$ are shown. The measure of size used is the rms pairwise separation

$$
\left\langle r_{i j}^{2}\right\rangle^{1 / 2}=\left[\frac{2}{N_{g}\left(N_{g}-1\right)} \sum_{i} \sum_{j<i} r_{i j}^{2}\right]^{1 / 2} .
$$

Groups in the DM models are smaller than their counterparts in the SP runs, due mainly to the work of dynamical friction. The dispersion in group sizes is greatly increased in the lowdensity DM run by the presence of many highly concentrated triplets. The $\Omega=1.0 \mathrm{DM}$ run is skewed less drastically toward smaller values of $\left\langle r_{i j}^{2}\right\rangle^{1 / 2}$.

The group velocity dispersions are defined in analogous fashion to equation (5) but are normalized to one-dimension. Note the relation between this pairwise definition and the usual observational definition, $\sigma_{\text {obs }}^{2}=\sum_{i}\left(\boldsymbol{v}_{i}-\boldsymbol{v}_{g}\right)^{2} /\left(N_{g}-1\right)$,

TABLE 1

Distribution of Galaxies among Groups

\begin{tabular}{|c|c|c|c|c|c|c|}
\hline Sample & Total & $N_{g}=1$ & $N_{g}=2$ & $N_{g}=3$ & $N_{g}=4$ & $N_{g}>4$ \\
\hline HG & 1208 & $314(0.26)$ & $168(0.14)$ & $123(0.10)$ & $104(0.09)$ & $499(0.41)$ \\
\hline $\mathrm{HG}^{\mathrm{a}} \ldots \ldots \ldots$ & 952 & $314(0.33)$ & $168(0.18)$ & $123(0.13)$ & $104(0.11)$ & $243(0.25)$ \\
\hline$\Omega=1.0, \mathrm{DM} \ldots$ & 800 & $278(0.35)$ & $132(0.17)$ & $63(0.08)$ & $48(0.06)$ & $279(0.35)$ \\
\hline$\Omega_{f}=0.15, \mathrm{DM}$ & 800 & $227(0.28)$ & $112(0.14)$ & $81(0.10)$ & $80(0.10)$ & $300(0.38)$ \\
\hline$\Omega=1.0, \mathrm{SP} \ldots \ldots \ldots \ldots$ & 800 & $238(0.30)$ & $136(0.17)$ & $81(0.10)$ & $72(0.09)$ & $273(0.34)$ \\
\hline$\Omega_{f}=0.15$, SP $\ldots \ldots \ldots$ & 800 & $231(0.29)$ & $138(0.17)$ & $75(0.09)$ & $64(0.08)$ & $292(0.37)$ \\
\hline
\end{tabular}

a Excludes Virgo (166 galaxies) and Ursa Major-Coma (90 galaxies). 


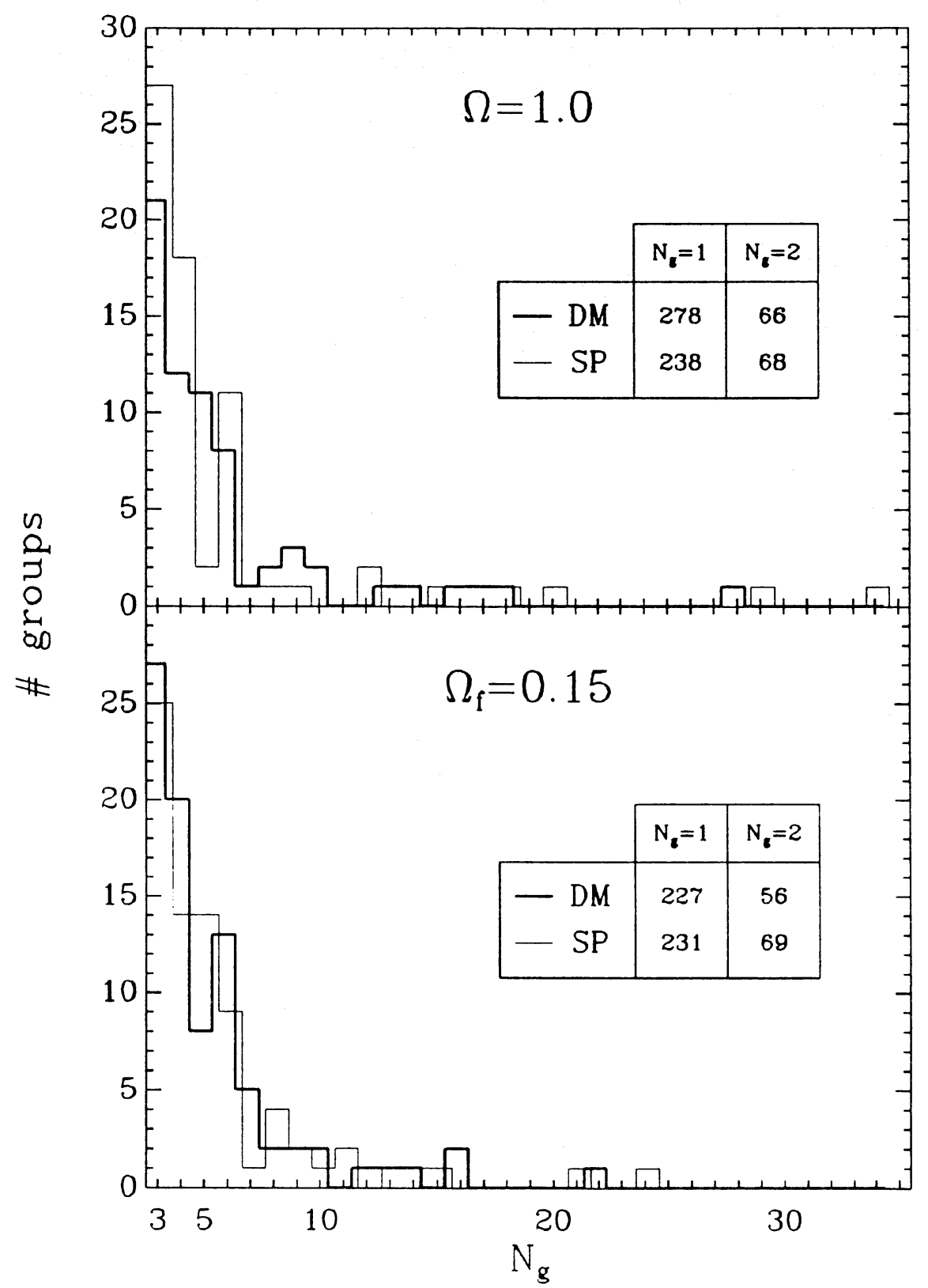

FIG. 1.-Multiplicity functions for each of the four models. Histograms for the dark matter (DM) and single-particle galaxy (SP) runs are offset slightly for clarity. Counts of binaries and singles are shown in inset.

measured relative to the group velocity $v_{g}$, is simply

$$
\left\langle v_{i j}^{2}\right\rangle^{1 / 2}=\left[\frac{2}{3 N_{g}\left(N_{g}-1\right)} \sum_{i} \sum_{j<i} v_{i j}^{2}\right]^{1 / 2}=\sqrt{2} \sigma_{\mathrm{obs}} .
$$

The most striking feature of the group velocities is the difference in magnitude between the two background cosmologies. Note the factor of 2 difference in the vertical scales for the left-hand sides of Figure 3. Also, the bin width used in the histogram for the low $\Omega$ models is half that used for $\Omega=1.0$. The scatter in velocities is smaller for groups in the darkmatter models, due to the much smoother mass distribution. The largest groups in the $\Omega=1.0 \mathrm{SP}$ run have dispersions in excess of $1000 \mathrm{~km} \mathrm{~s}^{-1}$. Rich clusters of galaxies have velocity dispersions approaching this scale, but the groups in this simu- lation contain only 30 or so galaxies. This is the usual difficulty encountered by simulations of critically dense universesvelocity dispersions generated in clusters are too large compared to observations.

The median values of group sizes and velocities are compared to the observational values of the HG sample in Table 2. The observational velocities have been multiplied by $(2)^{1 / 2}$, in accordance with equation (6). The simulated groups are more compact and have higher velocity dispersions than the observational groups. The observational projected cutoff of $r_{p}=$ $1.26 h_{s 0}^{-1} \mathrm{Mpc}$ will tend to pick out somewhat larger groups than those picked by the three-dimensional cutoff of $r_{c}=1$ Mpc used here, which corresponds roughly to a mean projected cutoff of $r_{p}=(\pi / 4) r_{c} \approx 0.8 \mathrm{Mpc}$. It is unlikely that selection effects alone are responsible for the discrepancy in group sizes, 


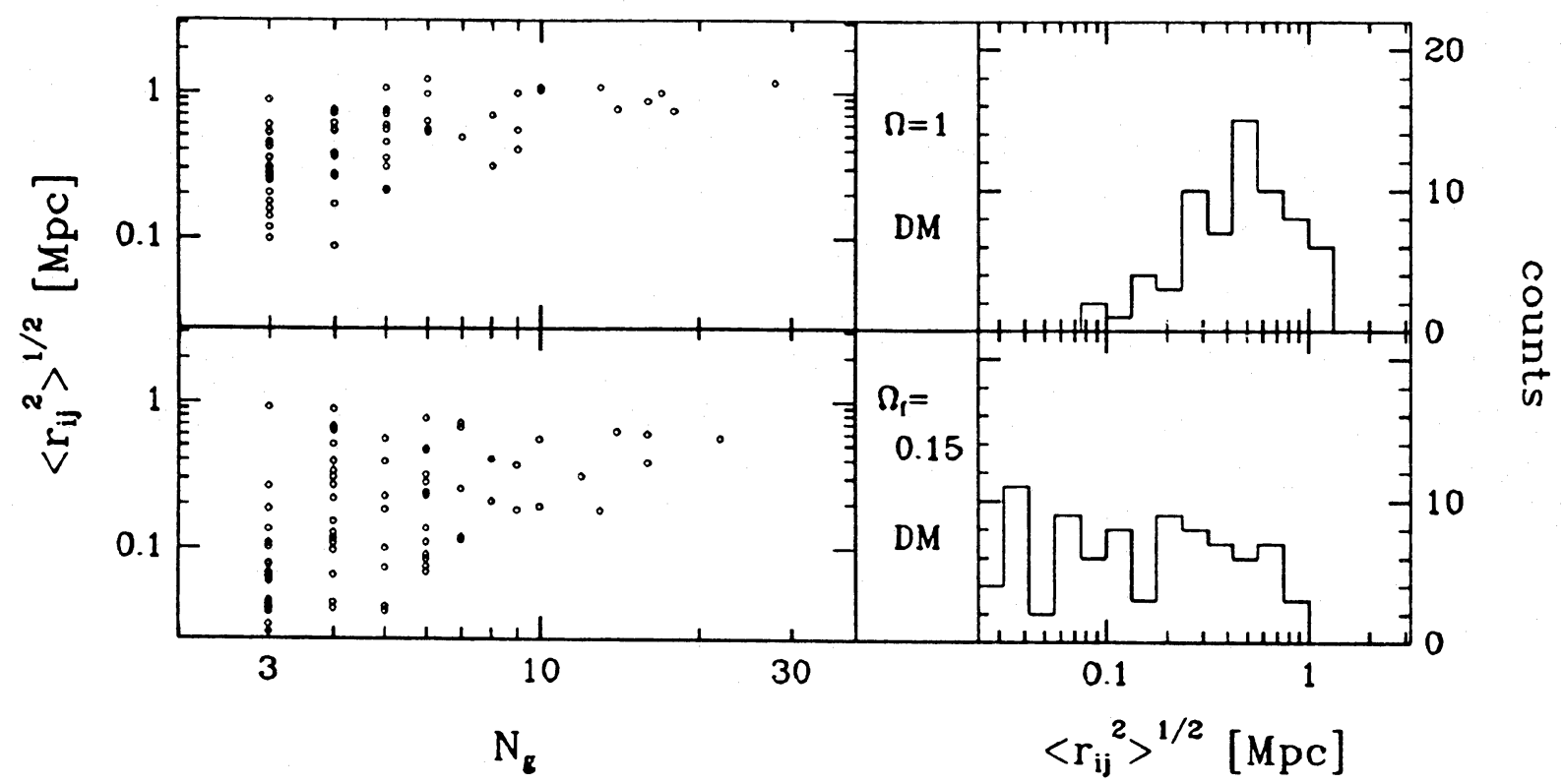

(a)

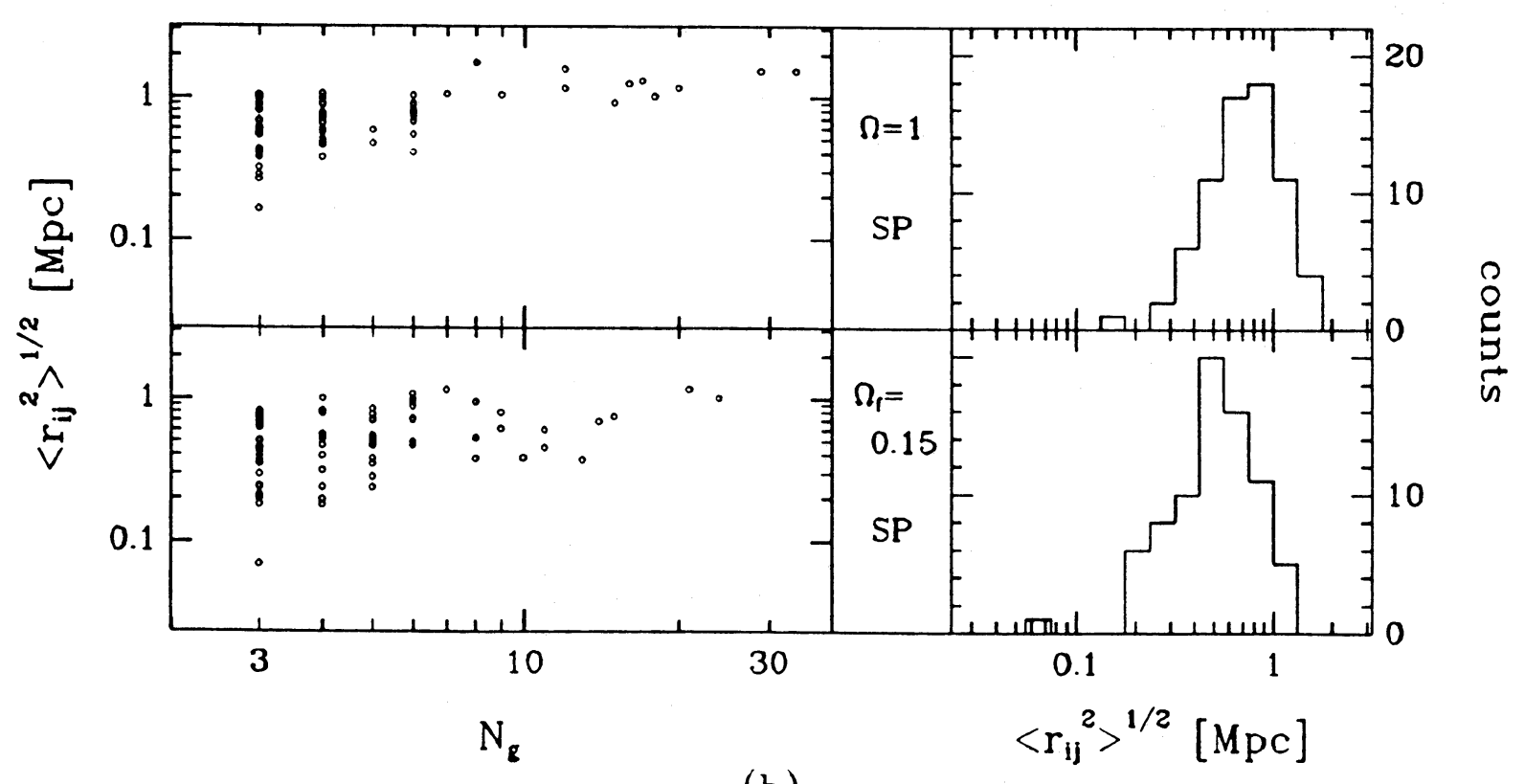

(b)

Fig. 2.-Scatter diagram and histogram of group sizes defined in eq. (5). (a) DM models $(b)$ SP models. Upper panels in both $(a)$ and $(b)$ are for $\Omega=1.0$, lower panels are $\Omega_{f}=0.15$.

TABLE 2

Median Group Properties

\begin{tabular}{|c|c|c|}
\hline Sample & $\begin{array}{l}\left\langle r_{i j}^{2}\right\rangle^{1 / 2} \\
(\mathrm{Mpc})\end{array}$ & $\begin{array}{c}\left\langle v_{i j}^{2}\right\rangle^{1 / 2} \\
\left(\mathrm{~km} \mathrm{~s}^{-1}\right)\end{array}$ \\
\hline HG .......... & $1.4^{\mathrm{a}}$ & 220 \\
\hline$\Omega=1.0, \mathrm{DM} .$. & 0.47 & 390 \\
\hline$\Omega_{f}=0.15, \mathrm{DM}$ & 0.14 & 230 \\
\hline$\Omega=1.0, \mathrm{SP} \ldots \ldots$ & 0.72 & 480 \\
\hline$\Omega_{f}=0.15, \mathrm{SP} \ldots$ & 0.51 & 290 \\
\hline
\end{tabular}

${ }^{\text {a }}$ Mean group size defined as $\left\langle r_{i j}\right\rangle=\left(4 V_{g} / \pi H_{0}\right) \sin \left[2 / N_{g}\left(N_{g}\right.\right.$ - 1) $\sum_{i} \sum_{j<i} \theta_{i j}$, where $\theta_{i j}$ is pairs' projected separation and $V_{g}^{g}$ is mean group velocity (scaled to $H_{0}=50 \mathrm{~km} \mathrm{~s}^{-1} \mathrm{Mpc}^{-1}$ ). especially for the $\Omega_{f}=0.15 \mathrm{DM}$ run. In Paper I, it was found that the small-scale effects of dynamical friction were unrealistic in this model - the distribution of binaries showed almost no pairs with separations between 80 and $1000 \mathrm{kpc}$. The same conclusion may be reached from the abnormally small group sizes. On the positive side, the centers of these extremely compact, low-velocity dispersion groups may be representative sites for the formation of central dominant galaxies.

The group velocity dispersions of the low-density models agree well with the observational data, the DM model more so than the run without dark halos. The $\Omega=1.0$ velocities are roughly a factor of 2 too large with or without dark halos. As 


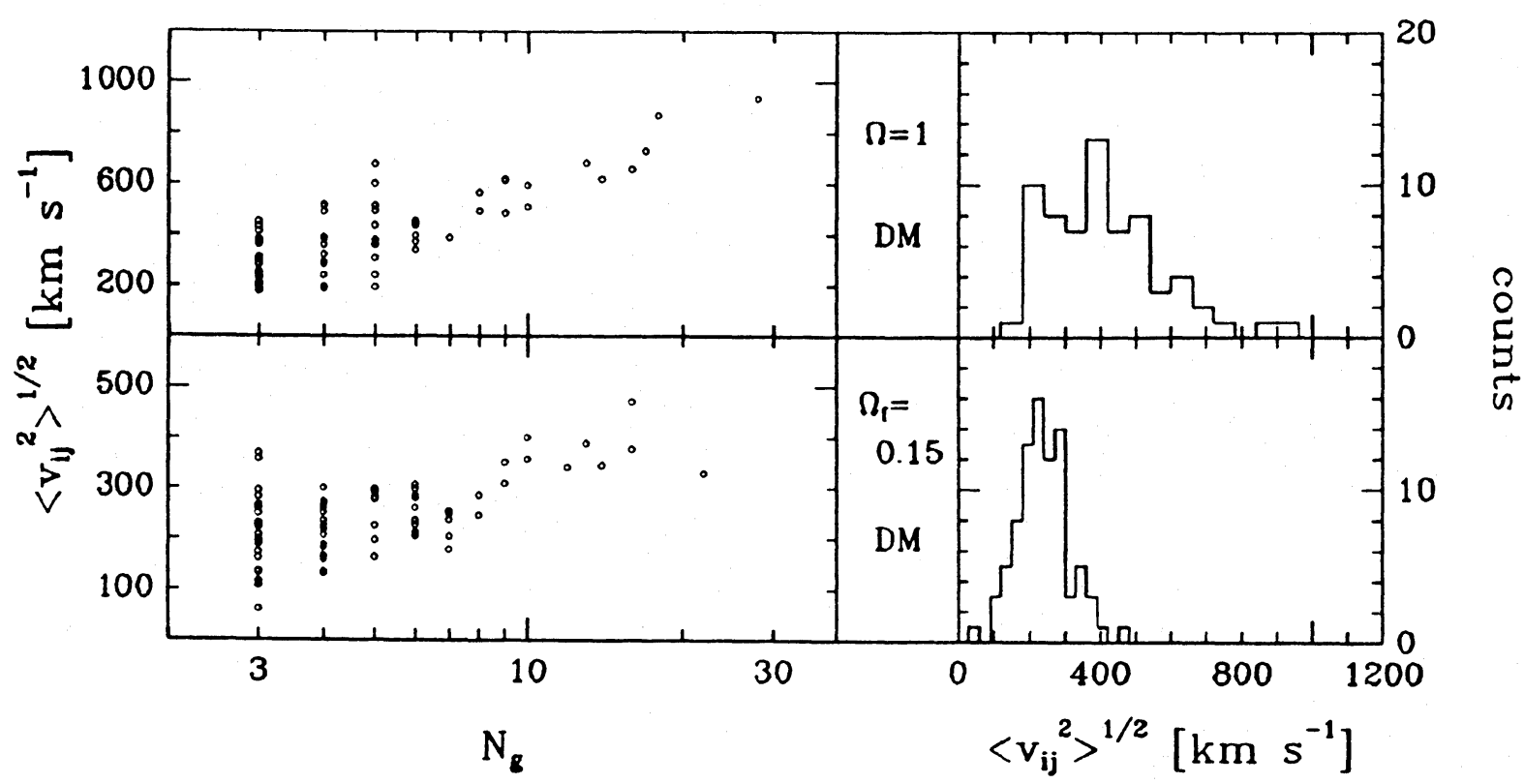

(a)

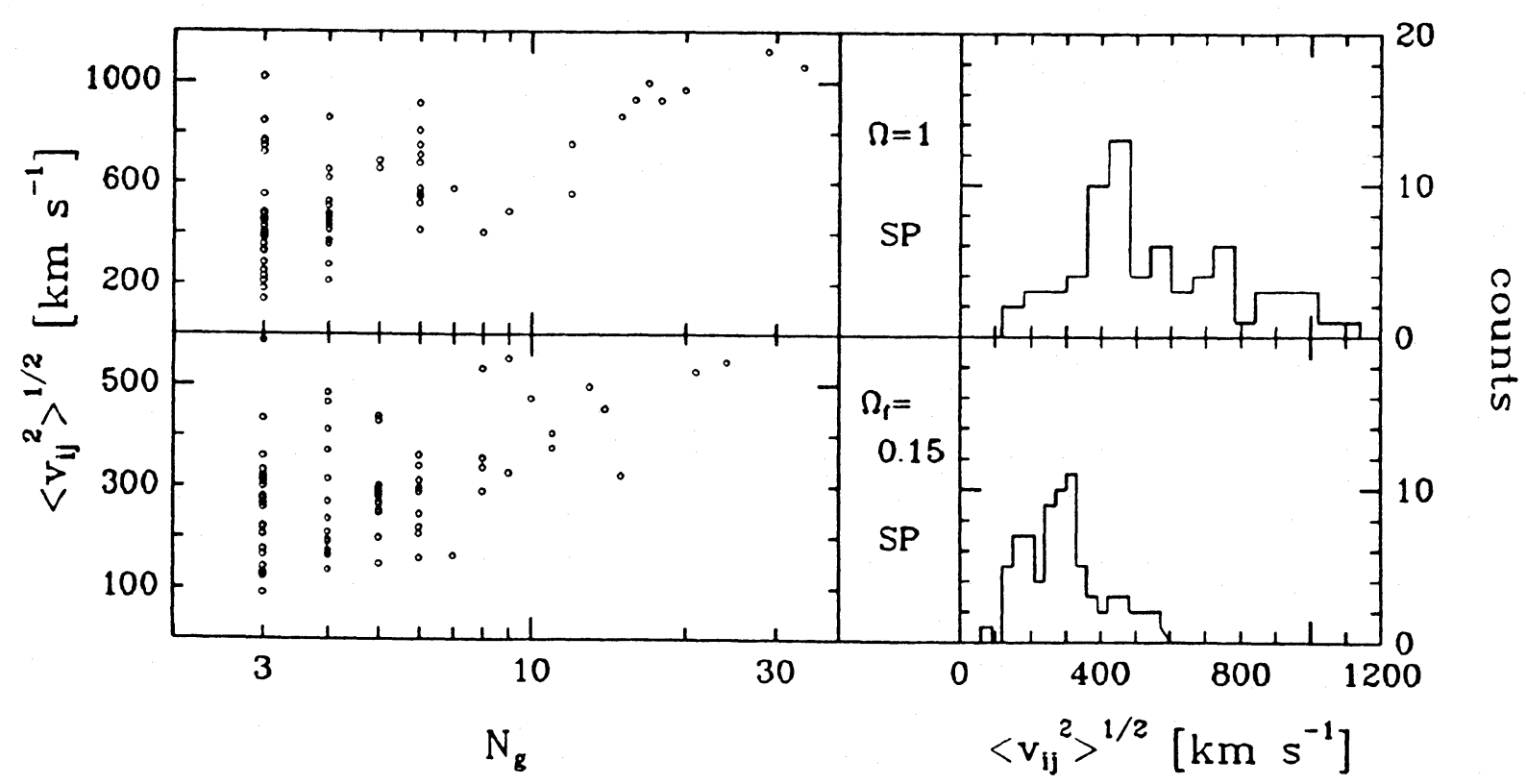

(b)

FIG. 3.-Scatter diagram and histogram of group velocity dispersions. Format is identical to Fig. (2). Note velocity scale in $\Omega_{f}=0.15$ models is a factor of 2 smaller than that used for $\Omega=1.0$. Also, the histogram bin width is half as big in the low-density runs, $30 \mathrm{~km} \mathrm{~s}^{-1} \mathrm{vs.} 60 \mathrm{~km} \mathrm{~s}^{-1}$ in the $\Omega=1.0$ models.

mentioned in Paper I, reduction of the $\Omega=1.0$ model velocities may require some form of large-scale biasing of the initial galaxy distribution to reduce the mean mass density in the vicinity of galaxies.

\section{GROUP MASS PROFILES}

Little is known observationally concerning the character of the mass distribution within groups of galaxies. That there exists dominant amounts of unseen matter within groups seems to be firmly established. To what degree is the dark matter bound in halos around galaxies or distributed uniformly throughout the group? How far beyond the visible edge of the group does it extend? How much gas exists in a typical group, and how is it distributed? Can accurate binding masses be derived from properties of the gas? These questions and many others are difficult problems to tackle from both an observational and theoretical perspective.

With the advent of X-ray observations of hot gas in groups of galaxies, it is now in principle possible, although in practice still difficult, to measure the binding (i.e., total) mass density as 
a function of distance from the group center. A study by Kriss, Cioffi, and Canizares (1983) of poor clusters with central dominant galaxies, cataloged by Albert, White, and Morgan (1977) and Morgan, Kayser, and White (1975), shows evidence for segregation of the luminous from the total mass. The luminosity profiles of these clusters are steeper than the total mass profiles. The mass-to-light ratio rises with distance from the group center, the integrated value being typical, $M / L \approx 100$, at radii comparable to the luminous edge of the group. Unfortunately, only a handful of such measurements have been made. More studies of this nature are needed on a wider variety of $X$-ray luminous groups to uncover systematic properties of the relative distributions of mass and light in clusters.

\section{a) The Density Run}

The mass distribution as a function of distance from the centroid of the galaxies was measured for all the groups in the simulated catalogs. In the SP runs, the centroid corresponds exactly to the group center of mass, since the galaxies identically trace the mass. The galaxies in the DM runs tend to sink to the centers of the local potential well generated by the total mass distribution, so their centroid will be very near the center of mass as well (see Figs. 3 and 10 of Paper I). Mean densities in logarithmic radial bins are obtained for sets of groups in specific membership categories. The procedure is equivalent to lumping together the mass distributions of groups within a given category to produce an "ensemble group," and measuring the ensemble's profile. Six ensembles are examinedgroups with $N_{g}=1,2,3$, and 4, along with $N_{g}=5$ or 6 , and finally, $N_{g}>6$.

Figure 4 shows the mean density profiles of groups in the dark-matter models, normalized to the current background mass density. The straight lines bracketing the data are power laws with index -2 (upper) and -3 (lower). The profiles are not pure power laws. The largest groups possess a reasonably well-resolved core which then rolls over into a near power-law profile. Cores in small $N_{g}$ groups are not as evident. The effective local power-law slopes, $n_{e}(r) \equiv d \log \rho / d \log r$, appear to span the range from $n_{e}(r)=-2$ to $n_{e}(r)=-3$, steepening from smaller to larger radii. Groups in the low-density model favor profiles somewhat steeper than those in the flat simulation, having $n_{e}(r) \geq-3$ outward of $100 \mathrm{kpc}$ from the centroid. The $\Omega_{f}=0.15$ groups are more isolated than their counterparts in the $\Omega=1.0$ model, where hierarchical clustering continues throughout the course of the run. The groups in the latter model experience larger external perturbations as well as continual infall. These effects will tend to prevent the outer profiles of groups from steepening.

How are the galaxies distributed among this mass? Figure 5 shows the galaxy overdensity profiles for groups with four or more members. The sizes of binaries and triplets are too small to generate meaningful radial profiles. The quantity measured, $\rho_{\text {lum }} / \rho_{b \text {, lum }}$, is the overdensity of galaxy counts within each bin relative to the background number density. Dispersions about the mean values shown are $\sim 0.5$ decades, or a factor of 3 , as can be judged from the scatter within the lines.

The central galaxy overdensities are factors of 3-5 times higher than the mass overdensities. The outer profiles are much steeper, having local power-law slopes $n_{e}(r) \approx-4$ in both models. For comparison, the SP model galaxy profiles are shown in Figure 6. The DM groups are more centrally concentrated, having higher central galactic overdensities and smaller core radii. The outer profiles appear slightly steeper in the DM models, but the differences are not significant given the degree of noise in these measurements.

The relative behavior of the luminous and total densities in the dark-matter models is qualitatively similar to the data for the poor clusters studied by Kriss, Cioffi and Canizares (1983). This may indicate that the same segregating mechanism is at work in both the real and simulated groups-i.e., that the simulations are correctly modeling the dynamical interaction between the luminous and dark components of real galaxy groups.

\section{b) True Mass-to-Light Ratios}

From the measured luminous and total mass profiles, the integrated mass-to-light ratio, $M / L(<r) \equiv M(<r) / M_{\text {lum }}(<r)$, is constructed for each group. The mean $M / L(<r)$ profiles are shown in Figure 7 for each of the different ensembles.

The cumulative $M / L$ 's show a rather remarkable universal relationship - a near power-law rise proportional to $r^{1 / 2}$ up to a value of $\sim 6$, followed by rollover and more gradual climb to the global value. The rollover occurs gradually at radii of $\sim 300 \mathrm{kpc}$ in the $\Omega_{f}=0.15$ model and $\sim 2 \mathrm{Mpc}$ in the $\Omega=1.0$ run. The identical galactic systems used initially prevent large mass-to light fluctuations from occurring on scales encompassing a few galaxies at the start of the calculation. This forces $M / L(<r)$ to the global value on scales larger than the typical group size. It is interesting that the dynamics below these scales cause the same relative degree of segregation to take place in binaries and triplets as occurs in the centers of groups with 10 or more members.

How much mass is contained within the volume of the group occupied by galaxies? Define $r_{*}$ to be the distance to the farthest galaxy in the group measured from the group centroid. Table 3 lists the median values of the cumulative mass-to-light ratios within $r_{*}\left(M L_{<r_{*}}\right), 2 r_{*}\left(M L_{<2 r_{*}}\right)$, and $4 r_{*}\left(M L_{<4 *}\right)$. The associated uncertainties are the ranges from the median to the upper and lower quartiles. The median values of $3.2(\Omega=1)$ and $3.6\left(\Omega_{f}=0.15\right)$ for $M L_{<r_{*}}$ indicate that the total amount of mass associated with galaxies in groups with three or more members amounts to $\sim 35 \%$ of the mass initially associated with them. The trend in $M L_{<r_{*}}$ with $N_{g}$ is easy to understand from examination of Figures 2 and 7 . Groups with more members are physically larger and thus sample more of the dark envelope than do smaller groups. The galaxies in binary and triplet systems have collected at the center of their dark halos-the amount of enclosed dark matter is no larger than the mass of the galaxies themselves.

\section{c) Rotation Curves}

Roughly $25 \%$ of the galaxies in the DM simulations have no neighbor within $1 \mathrm{Mpc}$ and fall into the $N_{g}=1$ group catagory. These "field" galaxies may be likened to isolated galaxies observed on the sky, most of which are spirals (Dressler 1984). One question that can be addressed in these simulations is whether or not flat rotation curves would be expected around such galaxies, where the circular velocity is measured by

$$
v_{\text {cir }}(r)=\sqrt{\frac{G M(r) r^{2}}{\left(r^{2}+\epsilon^{2}\right)^{3 / 2}}} .
$$

The limited dynamic range in the models precludes determination of rotation curves on scales under $30 \mathrm{kpc}$ - where nearly all observational measurements have been made-since $\epsilon=27$ 


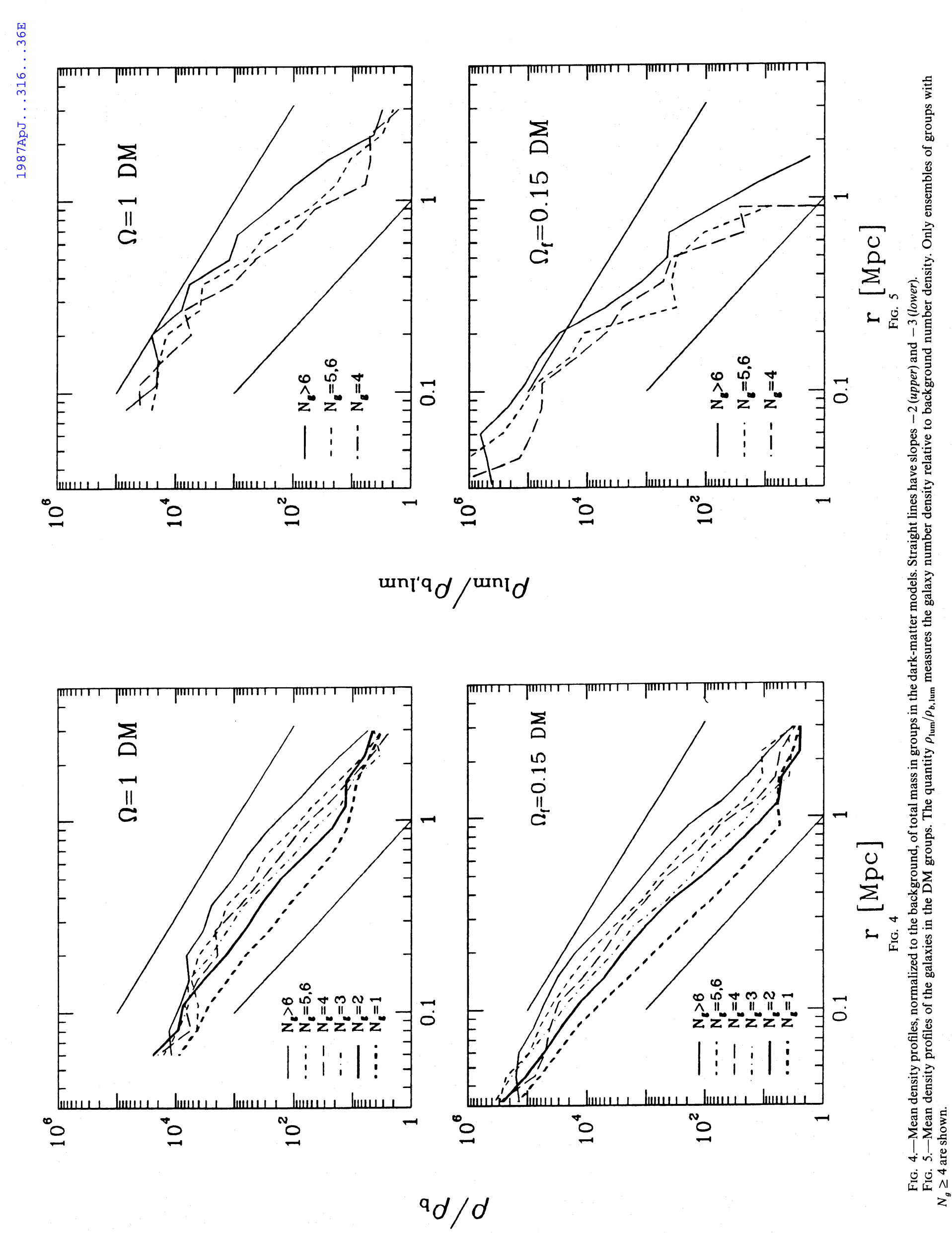




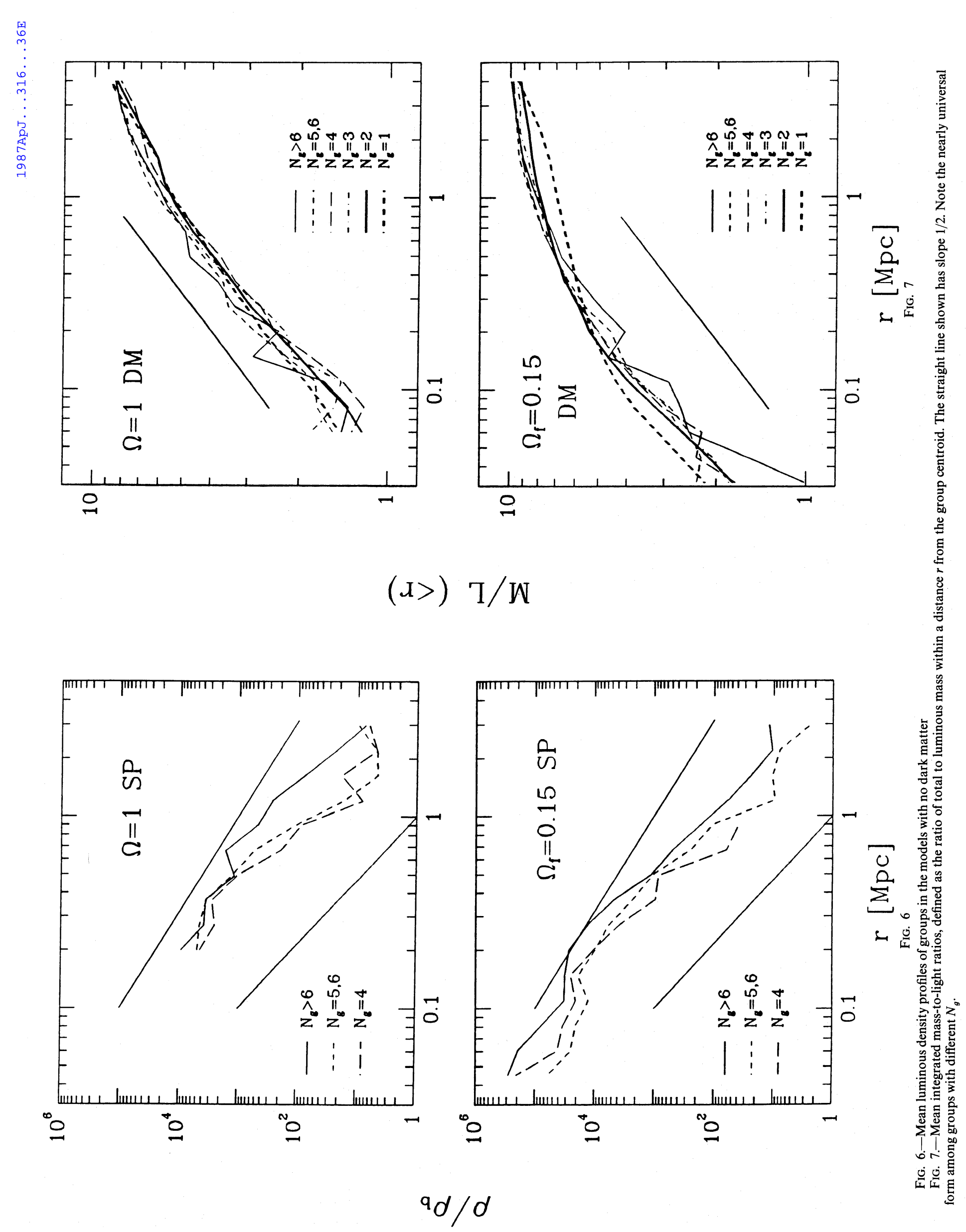


TABLE 3

Median Local $M / L$ Values

\begin{tabular}{|c|c|c|c|c|}
\hline Ensemble & $N$ & $\mathrm{ML}_{<r *}$ & $\mathrm{ML}_{<2 r *}$ & $\mathrm{ML}_{<4 r *}$ \\
\hline \multicolumn{5}{|c|}{$\Omega=1.0, \mathrm{DM}$} \\
\hline All $N_{g} \geq 3 \ldots$ & 66 & $3.2_{-1.1}^{+1.8}$ & $5.3_{-1.8}^{+2.0}$ & $6.7_{-1.6}^{+1.6}$ \\
\hline$N_{g}>6 \ldots \ldots$. & 14 & $5.7_{-1.5}^{+0.4}$ & $7.7_{-1.6}^{+0.8}$ & $8.4_{-1.1}^{+0.4}$ \\
\hline$N_{g}=5,6 \ldots$ & 19 & $4.0_{-0.9}^{+1.1}$ & $6.4_{-1.4}^{+1.5}$ & $7.4_{-1.1}^{+1.0}$ \\
\hline$N_{g}=4 \ldots \ldots \ldots \ldots \ldots$ & 12 & $2.9_{-1.1}^{+0.9}$ & $4.7_{-2.0}^{+1.0}$ & $6.1_{-1.8}^{+0.8}$ \\
\hline$N_{g}=3 \ldots \ldots \ldots \ldots \ldots$ & 21 & $2.1_{-0.4}^{+0.9}$ & $3.5_{-1.0}^{+1.6}$ & $5.1_{-1.1}^{+1.4}$ \\
\hline$N_{g}=2 \ldots \ldots \ldots \ldots \ldots$ & 66 & $1.4_{-0.2}^{+0.5}$ & $2.3_{-0.7}^{+1.4}$ & $3.2_{-1.0}^{+2.0}$ \\
\hline \multicolumn{5}{|c|}{$\Omega_{f}=0.15, \mathrm{DM}$} \\
\hline All $N_{g} \geq 3 \ldots \ldots \ldots \ldots$ & 85 & $3.6_{-1.8}^{+2.2}$ & $5.6_{-2.8}^{+2.8}$ & $6.9_{-2.3}^{+2.3}$ \\
\hline$N_{g}>6 \ldots \ldots \ldots \ldots \ldots$ & 17 & $6.2_{-1.4}^{+1.1}$ & $8.5_{-1.9}^{+1.9}$ & $9.2_{-0.8}^{+0.4}$ \\
\hline$N_{g}=5,6 \ldots \ldots \ldots \ldots$ & 21 & $4.3_{-2.1}^{+1.6}$ & $5.8_{-2.1}^{+2.7}$ & $7.8_{-2.1}^{+1.6}$ \\
\hline$N_{g}=4 \ldots \ldots \ldots \ldots \ldots$ & 20 & $5.0_{-2.2}^{+1.0}$ & $6.9_{-2.7}^{+1.5}$ & $7.5_{-1.7}^{+1.6}$ \\
\hline$N_{g}=3 \ldots \ldots \ldots \ldots \ldots \ldots$ & 27 & $1.7_{-0.4}^{+0.8}$ & $2.6_{-0.5}^{+1.7}$ & $4.4_{-1.3}^{+1.3}$ \\
\hline$N_{g}=2 \ldots \ldots \ldots \ldots \ldots$ & 56 & $1.2_{-0.2}^{+0.4}$ & $1.7_{-0.4}^{+1.3}$ & $2.8_{-0.8}^{+1.3}$ \\
\hline
\end{tabular}

$\mathrm{kpc}$ for $\Omega_{f}=0.15$ and $58 \mathrm{kpc}$ for $\Omega=1.0$. On scales outward of these distances, however, an accurate estimate of $v_{\text {cir }}$ can be made. Figure 8 shows the rotation curves obtained for the ensemble of isolated galaxies in each model. The data and error bars are the mean $v_{\text {cir }}$ and standard deviation among the galaxies. The mean density profile around isolated galaxies is very nearly isothermal in the range shown for the $\Omega=1.0$ model (see Fig. 4). Thus, a flat rotation curve results. In the low-density universe, the density is close to isothermal within the inner $100 \mathrm{kpc}$ but steepens outward of this scale. The circular velocity is flat between 50 and $100 \mathrm{kpc}$, then drops in a roughly linear fashion from a value of $235 \mathrm{~km} \mathrm{~s}^{-1}$ at $100 \mathrm{kpc}$ to
$175 \mathrm{~km} \mathrm{~s}^{-1}$ at $250 \mathrm{kpc}$. The $\Omega=1.0$ rotation velocities are in good agreement with those found by Frenk et al. (1985), who modeled the growth of structure on scales containing a few galaxies in a universe dominated by cold dark matter. The rotation curves for the 10 most massive objects grown in their simulation were flat from $\sim 50$ to $200 \mathrm{kpc}$, with $v_{\text {cir }}$ ranging in value from $130 \mathrm{~km} \mathrm{~s}^{-1}$ to $400 \mathrm{~km} \mathrm{~s}^{-1}$.

The magnitude of the rotation velocity in the $\Omega=1.0$ model, $325 \mathrm{~km} \mathrm{~s}^{-1}$, is larger than typical observed values of $200 \mathrm{~km}$ $\mathrm{s}^{-1}$ (Bahcall and Casertano 1985). If the real universe is flat, $\Omega_{0}=1$, this result indicates that it would be difficult for dark halos around bright galaxies to contain all the missing mass in the universe (see also conclusions of Paper I).

\section{VIRIAL MASS ESTIMATES}

The analysis in the previous section presented evidence that galaxies are not fair tracers of the full mass distribution in groups dominated by dark matter. Rather, the heavier luminous particles tend to pile up at the center of the enveloping dark-matter distribution. The bias which this segregation introduces into dynamical mass estimates is addressed in this section.

\section{a) Forms of the Virial Theorem}

The virial theorem has historically been the workhorse used to estimate the masses of clusters of galaxies. Application to individual groups is problematic because instantaneous phase space sampling need not accurately reflect the time-averaged quantities required in the formal definition (given below). This lack of ergodicity gives rise to a wide dispersion in virial mass estimates (Turner et al. 1979), which can exist even for a set of well-defined identical systems (Heisler, Tremaine, and Bahcall 1985). However this source of error is small when dealing with

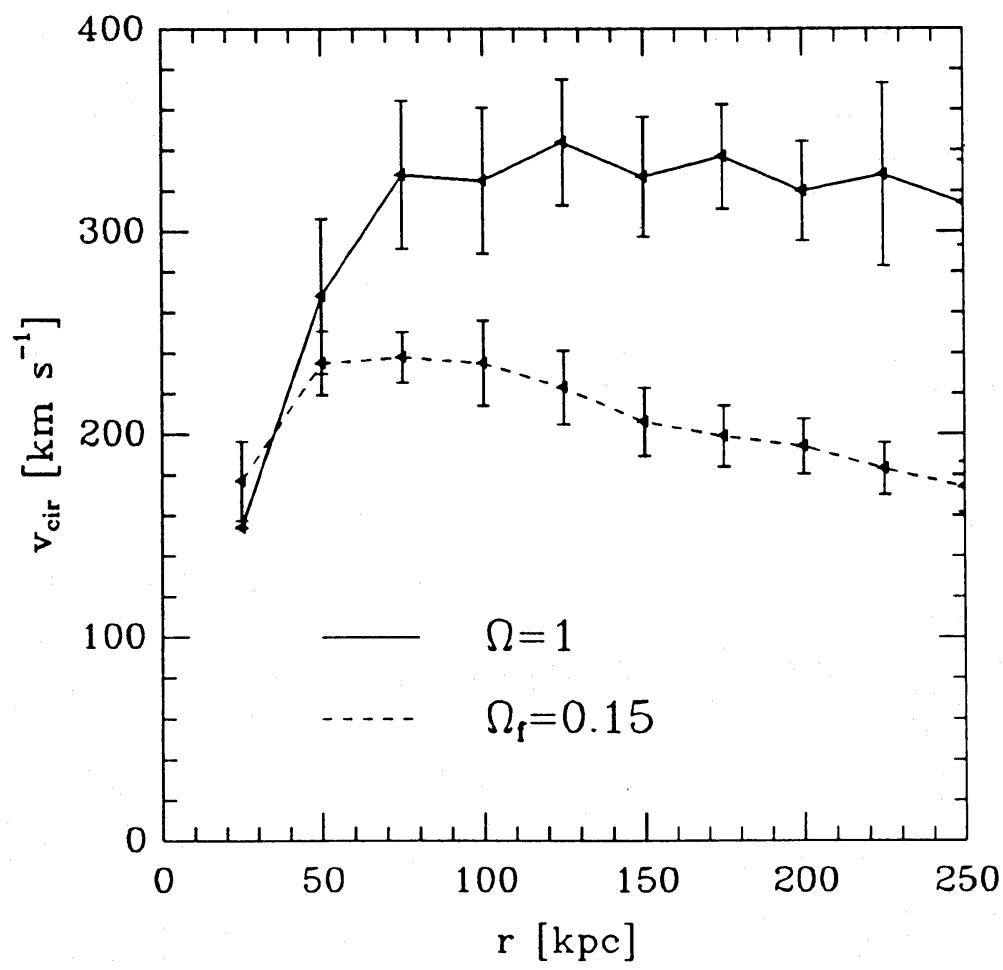

FIG. 8.-Rotation velocity, $v_{\text {cir }}^{2}(r)=G M(r) r^{2} /\left(r^{3}+\epsilon^{2}\right)^{3 / 2}$, for isolated galaxies in the dark-matter models. These galaxies have no neighbors within 1 Mpc. Means and standard deviations of the data in each bin are shown. 
sufficiently large groups ( $\sim 10$ or more members), or when median values from an ensemble of groups are used.

Following the usual derivation of the virial theorem, consider a system of $N$-bodies, each of mass $m$. Take the second time derivative of the quantity $I=\frac{1}{2} m \sum_{i}\left|\boldsymbol{r}_{i}\right|^{2}$, where $\boldsymbol{r}_{i}$ is measured from the system's center of mass,

$$
\begin{aligned}
\frac{d}{d t^{2}} I & =\sum_{i} m\left|\frac{d}{d t} \boldsymbol{r}_{i}\right|^{2}+\sum_{i} m r_{i} \cdot \frac{d}{d t^{2}} \boldsymbol{r}_{i} \\
& =\sum_{i} m v_{i}^{2}+\sum_{i} m r_{i} \cdot g_{i} .
\end{aligned}
$$

If we are dealing with an isolated system of point masses in steady state equilibrium, then the time average of the left-hand side of equation (8) is zero, and we are led to the standard virial estimate of the group mass

$$
M=\left(\frac{N}{G}\right) \frac{\sum_{i} v_{i}^{2}}{\sum_{i} \sum_{j<i} 1 / r_{i j}} .
$$

The particles in the simulations interact via a "softened" point mass interaction, $g_{i j}=-G m r_{i j} /\left(r_{i j}^{2}+\epsilon^{2}\right)^{3 / 2}$. This changes the form of the standard mass estimate, equation (9) to

$$
M=\left(\frac{N}{G}\right) \frac{\sum_{i} v_{i}^{2}}{\sum_{i} \sum_{j<i} r_{i j}^{2} /\left(r_{i j}^{2}+\epsilon^{2}\right)^{3 / 2}} .
$$

This mass estimate will differ appreciably from equation (9) only if there are significant numbers of pairs in the group with separation comparable to to $\epsilon$. From Figure $2 a$, it is evident that this is the case for small $N_{g}$ groups in the dark matter simulations. Note the radically different limiting behavior of the two estimators, equations (9) and (10), as $r_{i j} \rightarrow 0$. The standard mass estimate goes to 0 linearly with $r_{i j}$ while the softened form diverges as $r_{i j}^{-2}$.

In the two-component models, the presence of dark matter implies that the acceleration of a given galaxy is not given by a simple sum over the other galaxies in the group. The virial theorem will still hold if the two-component system of particles is in an equilibrium state. To test this, a pairwise form of the virial theorem is useful

$$
M=N \frac{\sum_{i} \sum_{j<i} v_{i j}^{2}}{\sum_{i} \sum_{j<i}-r_{i j} \cdot g_{i j}^{\mathrm{tot}}} .
$$

Here $\boldsymbol{g}_{i j}^{\text {tot }}$ is the difference in the total accelerations between luminous particles $i$ and $j$ which, for the discrete systems simulated in the models, is

$$
\begin{aligned}
\boldsymbol{g}_{i j}^{\mathrm{tot}}=-2 \frac{G m_{\mathrm{lum}} \boldsymbol{r}_{i j}}{\left(r_{i j}^{2}+\epsilon^{2}\right)^{3 / 2}} & -\sum_{\substack{\text { lum } \\
k \neq i, j}}\left[\frac{G m_{\mathrm{lum}} \boldsymbol{r}_{i k}}{\left(r_{i k}^{2}+\epsilon^{2}\right)^{3 / 2}}-\frac{G m_{\mathrm{lum}} \boldsymbol{r}_{j k}}{\left(r_{j k}^{2}+\epsilon^{2}\right)^{3 / 2}}\right] \\
& -\sum_{\substack{\mathrm{dark} \\
n}}\left[\frac{G m_{\mathrm{dark}} \boldsymbol{r}_{i n}}{\left(r_{i n}^{2}+\epsilon^{2}\right)^{3 / 2}}-\frac{G m_{\mathrm{dark}} \boldsymbol{r}_{j n}}{\left(r_{j n}^{2}+\epsilon^{2}\right)^{3 / 2}}\right],
\end{aligned}
$$

which is a sum over all particles present, both luminous and dark. Equation (11) provides a true indication of whether or not the two-component clusters are in virial equilibrium. It uses full knowledge of the dark-matter distribution, unlike equations (9) and (10), which are based solely on the kinematic data of the galaxies.

Finally, observational virial analysis is done in projected space. If the galaxies' positions and velocities are randomly oriented with respect to the observer's direction, then the stan- dard mass estimate takes the projected form

$$
M=\left(\frac{3 \pi N}{2 G}\right) \frac{\sum_{i} v_{\mathrm{los}, i}^{2}}{\sum_{i} \sum_{j<i} 1 / r_{p, i j}},
$$

where $r_{p}$ is the projected pair separation on the sky and $v_{\text {los }}$ is the velocity along the line of sight.

\section{b) Virial Analysis}

Mass estimates based on equations (9), (10), and (13) were calculated from the positions and velocities of the galaxies within each group. Dividing the estimated masses by the known luminous mass within the group yields mass-to-light ratios. The median and quartile values of these ratios for groups in different membership ensembles are given in Tables 4 and 5 for the DM and SP runs, respectively. The quantity $\mathrm{ML}_{\mathrm{vT}}$ is the mass-to-light ratio calculated from the standard virial theorem, equation (9), $M L_{\mathrm{VTs}}$ is that obtained from the softened form, equation (10), and $\mathrm{ML}_{P i}$ uses the projected mass estimate, equation (13), along the $i$-axis, and $i=x, y, z$ are each measured. Also given is the quantity $M L_{\mathrm{RDG}}$, which uses equation (11) and the total acceleration, equation (12), for which the known mass and positions of both the galaxies and the darkmatter particles (in the DM runs) are used. An accurate indicator of the total mass will yield a median $M / L$ equal to the global mean value, $M / L_{\text {glob }}=10$ in the DM models and $M / L_{\text {glob }}=1$ in the SP runs.

Consider first the data for the runs with no dark matter, Table 5. The values of $M L_{R D G}$ and $M L_{V T s}$ are identical because the total acceleration is given exactly by a sum over neighboring galaxies. The accuracy of these estimates is excellent, the median for all groups with $N_{g} \geq 3$ identically matches the global value, with a scatter measured by the quartiles of $\sim 30 \%$. The median standard values, $\mathrm{ML}_{\mathrm{VT}}$, underestimate the

\begin{tabular}{|c|c|c|c|c|c|c|c|}
\hline Ensemble & $N$ & $\mathrm{ML}_{\mathrm{RDG}}$ & $\mathrm{ML}_{\mathrm{VT}}$ & $\mathrm{ML}_{\text {vTS }}$ & $\mathrm{ML}_{P x}$ & $\mathrm{ML}_{P y}$ & $\mathrm{ML}_{P z}$ \\
\hline \multicolumn{8}{|c|}{$\Omega=1.0, \mathrm{DM}$} \\
\hline All $N_{g} \geq 3 \ldots$ & 66 & $11.3_{-3.9}^{+5.9}$ & $2.2_{-0.9}^{+1.9}$ & $3.5_{-1.7}^{+1.8}$ & 1.8 & 2.5 & 1.9 \\
\hline$N_{g}>6 \ldots \ldots \ldots$ & 14 & $12.9_{-2.2}^{+2.3}$ & $3.9_{-1.8}^{+1.5}$ & $5.4_{-0.8}^{+2.3}$ & 3.8 & 3.2 & 3.6 \\
\hline$N_{g}=5,6 \ldots \ldots$ & 19 & $10.1_{-2.1}^{+8.1}$ & $2.8_{-1.3}^{+0.9}$ & $3.7_{-1.2}^{+1.2}$ & 1.8 & 2.9 & 2.7 \\
\hline$N_{g}=4 \ldots \ldots \ldots$ & 12 & $9.0_{-2.9}^{+5.8}$ & $1.6_{-0.5}^{+0.9}$ & $2.8_{-1.5}^{+1.9}$ & 1.4 & 1.8 & 1.3 \\
\hline$N_{g}=3 \ldots \ldots \ldots$ & 21 & $10.7_{-5.1}^{+10.3}$ & $1.6_{-0.7}^{+1.3}$ & $2.6_{-1.3}^{+1.3}$ & 1.0 & 0.9 & 1.2 \\
\hline$N_{g}=2 \ldots \ldots$ & 66 & $8.6_{-4.9}^{+12.4}$ & $1.9_{-1.2}^{+1.4}$ & $2.5_{-1.5}^{+2.2}$ & 1.7 & 0.7 & 1.2 \\
\hline$N_{g}=2^{\mathrm{a}} \ldots$ & 66 & 15.1 & 2.2 & 4.2 & $\cdots$ & $\cdots$ & $\cdots$ \\
\hline \multicolumn{8}{|c|}{$\Omega_{f}=0.15, \mathrm{DM}$} \\
\hline All $N_{g} \geq 3$ & 85 & $8.9_{-4.3}^{+6.7}$ & $1.3_{-0.5}^{+0.9}$ & $2.8_{-0.9}^{+1.2}$ & 1.1 & 1.0 & 1.5 \\
\hline$N_{g}>6 \ldots \ldots \ldots$ & 17 & $11.0_{-4.9}^{+4.6}$ & $1.5_{-0.3}^{+0.9}$ & $3.0_{-0.9}^{+0.9}$ & 1.6 & 1.4 & 2.0 \\
\hline$N_{g}=5,6 \ldots \ldots$ & 21 & $8.5_{-3.6}^{+6.3}$ & $1.3_{-0.4}^{+1.1}$ & $2.9_{-1.1}^{+0.6}$ & 1.3 & 1.3 & 1.2 \\
\hline$N_{g}=4 \ldots \ldots \ldots$ & 20 & $5.4_{-6.6}^{+8.0}$ & $1.0_{-0.4}^{+1.9}$ & $2.6_{-0.7}^{+3.5}$ & 1.1 & 0.7 & 1.3 \\
\hline$N_{g}=3 \ldots$ & 27 & $9.8_{-5.5}^{+8.0}$ & $1.1_{-0.6}^{+0.6}$ & $2.6_{-1.4}^{+1.3}$ & 0.8 & 0.5 & 1.6 \\
\hline$N_{g}=2 \ldots \ldots \ldots$ & 56 & $7.5_{-5.3}^{+10.2}$ & $0.7_{-0.3}^{+1.0}$ & $2.1_{-1.3}^{+2.8}$ & 0.5 & 0.5 & 0.4 \\
\hline$N_{g}=2^{\mathrm{a}} \ldots \ldots \ldots$ & 56 & 15.9 & 0.8 & 2.7 & $\ldots$ & $\ldots$ & $\cdots$ \\
\hline
\end{tabular}
global value by small amounts, $10 \%(\Omega=1.0)$ and $20 \%\left(\Omega_{f}=\right.$ 0.15 ), because a small fraction of pairs have separations comparable to the particle softening.

The accuracies within individual membership classes are also quite good for any of these three measures, except for the

TABLE 4

Median Dynamical $M / L$ Estimates with Dark Matter

${ }^{\text {a }}$ Using ensemble virial estimate, eq. (15) 
TABLE 5

Median Dynamical $M / L$ Estimates without Dark Matter

\begin{tabular}{|c|c|c|c|c|c|c|c|}
\hline Ensemble & $N$ & $\mathrm{ML}_{\text {RDG }}$ & $\mathrm{ML}_{\mathrm{VT}}$ & $\mathrm{ML}_{\mathbf{v T s}}$ & $\mathrm{ML}_{P x}$ & $\mathrm{ML}_{P y}$ & $\mathrm{ML}_{P z}$ \\
\hline \multicolumn{8}{|c|}{$\Omega=1.0, \mathrm{SP}$} \\
\hline All $N_{g} \geq 3 \ldots$. & 70 & $1.0_{-0.3}^{+0.4}$ & $0.9_{-0.3}^{+0.3}$ & $1.0_{-0.3}^{+0.4}$ & 0.9 & 0.7 & 0.9 \\
\hline$N_{g}>6 \ldots \ldots \ldots$ & 12 & $1.1_{-0.2}^{+0.1}$ & $1.0_{-0.2}^{+0.2}$ & $1.1_{-0.1}^{+0.1}$ & 1.1 & 0.9 & 0.9 \\
\hline$N_{g}=5,6 \ldots \ldots$ & 13 & $1.2_{-0.3}^{+0.2}$ & $1.0_{-0.2}^{+0.2}$ & $1.2_{-0.3}^{+0.2}$ & 0.9 & 0.7 & 1.8 \\
\hline$N_{g}=4 \ldots \ldots \ldots$ & 18 & $0.8_{-0.3}^{+0.5}$ & $0.8_{-0.3}^{+0.4}$ & $0.8_{-0.3}^{+0.5}$ & 1.3 & 0.6 & 0.8 \\
\hline$N_{g}=3 \ldots \ldots \ldots$ & 27 & $0.9_{-0.3}^{+0.7}$ & $0.9_{-0.3}^{+0.5}$ & $0.9_{-0.3}^{+0.7}$ & 0.6 & 0.6 & 0.7 \\
\hline$N_{g}=2 \ldots \ldots \ldots$ & 68 & $0.6_{-0.4}^{+0.6}$ & $0.6_{-0.3}^{+0.5}$ & $0.6_{-0.4}^{+0.6}$ & 0.5 & 0.3 & 0.2 \\
\hline$N_{g}=2^{\mathrm{a}} \ldots$ & 68 & 1.0 & 0.8 & 1.0 & $\cdots$ & $\cdots$ & $\cdots$ \\
\hline \multicolumn{8}{|c|}{$\Omega_{f}=0.15, \mathrm{SP}$} \\
\hline All $N_{g} \geq 3 \ldots$. & 77 & $1.0_{-0.3}^{+0.3}$ & $0.8_{-0.3}^{+0.2}$ & $1.0_{-0.3}^{+0.3}$ & 0.7 & 0.7 & 0.7 \\
\hline$N_{g}>6 \ldots \ldots \ldots$ & 15 & $1.0_{-0.1}^{+0.3}$ & $0.9_{-0.1}^{+0.2}$ & $1.0_{-0.1}^{+0.3}$ & 0.9 & 0.8 & 0.9 \\
\hline$N_{g}=5,6 \ldots \ldots$ & 23 & $0.9_{-0.2}^{+0.2}$ & $0.8_{-0.2}^{+0.2}$ & $0.9_{-0.2}^{+0.2}$ & 0.5 & 0.7 & 0.7 \\
\hline$N_{g}=4 \ldots \ldots \ldots$ & 14 & $0.8_{-0.3}^{+0.6}$ & $0.8_{-0.3}^{+0.6}$ & $0.8_{-0.3}^{+0.6}$ & 0.9 & 0.5 & 0.9 \\
\hline$N_{g}=3 \ldots \ldots \ldots$ & 25 & $1.0_{-0.4}^{+0.4}$ & $0.9_{-0.4}^{+0.2}$ & $1.0_{-0.4}^{+0.4}$ & 0.6 & 0.7 & 0.5 \\
\hline$N_{g}=2 \ldots \ldots \ldots$ & 69 & $0.4_{-0.3}^{+0.6}$ & $0.4_{-0.3}^{+0.4}$ & $0.4_{-0.3}^{+0.6}$ & 0.3 & 0.2 & 0.1 \\
\hline$N_{g}=2^{\mathrm{a}} \ldots \ldots \ldots$ & 69 & 1.1 & 0.6 & 1.1 & $\cdots$ & $\cdots$ & $\cdots$ \\
\hline
\end{tabular}

a Using ensemble virial estimate, eq. (17).

case of binaries. The reason for this discrepancy is rooted in the eccentric nature of the binary orbits and in the assumption of ergodicity - the replacement of an ensemble average for a time average. A pair of point masses bound in an orbit of eccentricity $\epsilon$ spends most of the time near apocenter, where the virial ratio is

$$
m_{\mathrm{est}}=\left(v_{1}^{2}+v_{2}^{2}\right) r_{12} \approx m_{\mathrm{true}}(1-e) .
$$

Binary orbits in the point-mass models of Efstathiou and Eastwood (1981) were found to be highly eccentric (Evrard and Yahil 1985a), and the eccentricities of binaries in the SP models should be similar. With most of these pairs found near apocenter, the use of the median mass estimate is likely to result in an underestimate. A more reliable indicator is the use of the ensemble ratio

$$
m_{\text {est }}^{*}=\frac{\sum_{\text {groups }}\left(v_{1}^{2}+v_{2}^{2}\right)}{\sum_{\text {groups }}\left(1 / r_{12}\right)} .
$$

The results of using this ratio for the ensemble of binaries is shown in the row labeled $N_{g}=2^{*}$. For the SP runs, this is accurate to within $10 \%$. The use of equation (15) can be extended to groups with $N_{g}>2$, but the results do not differ significantly from those obtained using the median.

From Table 5, it is clear that the median projected values, $\mathrm{ML}_{P i}$, generally agree well with the three-dimensional $M L_{\mathrm{VT}}$ for groups with four or more members. For triplets and binaries, projected values tend to be lower than the threedimensional estimates due to undersampling of velocity space, as noted by Heisler, Tremaine, and Bahcall (1985). The sum $\sum_{i} v_{\text {los, } i}^{2} / \sigma_{g}^{2}$ follows a $\chi^{2}$ distribution with $N_{g}-1$ degrees of freedom. For triplets, the probability of underestimating the actual group velocity dispersion, $\sigma_{g}$, by a factor of 2 is $\sim 0.32$. The probability for quartets is only $8 \%$.

Consider now the results for the dark-matter models given in Table 4. Evidently, the two-component groups are very nearly in virial equilibrium, as witnessed by the median values of $\mathrm{ML}_{\mathbf{R D G}}$. The values for all groups with three or more members are accurate to within $\sim 10 \%$ of $M / L_{\text {glob }}$. The scatter as measured by quartiles is somewhat larger than that found in the SP runs, being $\sim 50 \%$. The largest discrepancy for an individual membership class is a factor of 2 underestimate for groups with 4 members in the $\Omega_{f}=0.15$ run.

Substantial errors arise when the standard form, $\mathrm{ML}_{\mathrm{VT}}$, is applied to the two-component models. In the $\Omega_{f}=0.15$ model, $\mathrm{ML}_{\mathrm{VT}}$ underestimates $M / L_{\text {glob }}$ by a full order of magnitude for small groups and a factor of 7 for the largest groups. The discrepancies are smaller in the $\Omega=1.0$ run, ranging from a factor of 6 underestimate in small groups to a factor of $\sim 3$ for the largest. For all groups with $N_{g} \geq 3, M / L_{\text {glob }}$ is underestimated by factors of $10 / 2.2 \approx 5(\Omega=1.0)$ and $10 / 1.3 \approx 8\left(\Omega_{f}=\right.$ $0.15)$.

Table 6 summarizes the results of estimating $\Omega$ using equation (2) with the median virial $M / L$ 's for groups with three or more members. The standard version yields estimates of $\Omega=0.22$ and $\Omega=0.02$ for the $\Omega=1.0$ and $\Omega_{f}=0.15$ models, respectively, with statistical errors as determined by the quartiles of less than a factor of 2 . Some of this bias is removed when the particle softening is taken into account. Still, the underestimate when compared to the global value remains substantial.

The softened virial mass estimates for groups with three or more members do measure to $\sim 20 \%$ accuracy the amount of mass in the local vicinity of galaxies, as comparison of $\mathrm{ML}_{\mathrm{VTS}}$ in Table 4 with $\mathrm{ML}_{<r *}$ in Table 3 indicates. The median virial mass-to-light ratio of observed groups is $\sim 20 \%$ of the critical value given in equation (1). If galaxies are not as "soft" as the particles in the models, so that a pointlike potential is applicable, then the model results suggest this mass-to-light ratio is measuring only the amount of mass within the luminous parts of groups. If galaxies formed within extended dark halos, it is quite possible that enough mass to make $\Omega_{0}=1$ could be hidden in dark envelopes extending beyond the regions of clustered luminous matter.

If the real universe is flat, however, there remains the problem of reconciling observed galactic velocities with those in the $\Omega=1$ models, which are roughly twice as large. Solution to this problem may require initial biasing of the galaxy distribution on scales larger than the mean intergalactic separation. The effect of the bias will be to reduce the depths of the potential wells through which galaxies must fall, thereby lowering their thermal velocities. At present, arguments in favor of biased galaxy formation are rather crude, but candidate physical mechanisms have been presented (Dekel and Rees 1986).

\section{CONCLUSIONS}

Properties of groups of galaxies in the large-scale clustering simulations introduced in Paper I were examined. A friends of friends algorithm was applied to the models, and the resultant group catalogs compared to the observational catalog of Huchra and Geller (1982). Mass and luminosity profiles were

TABLE 6

\begin{tabular}{|c|c|c|}
\hline Virial Form & $\Omega=1.0$ & $\Omega_{f}=0.15$ \\
\hline $\mathrm{ML}_{\mathrm{VT}} \ldots \ldots \ldots \ldots \ldots \ldots \ldots$ & 0.22 & 0.02 \\
\hline $\mathrm{ML}_{\mathbf{v T S}} \ldots \ldots \ldots \ldots \ldots \ldots \ldots$ & 0.35 & 0.04 \\
\hline $\mathrm{ML}_{\mathrm{RDG}} \ldots \ldots \ldots \ldots \ldots \ldots \ldots$ & 1.1 & 0.13 \\
\hline
\end{tabular}

EstiMATES OF $\Omega$ IN DM MODELS ${ }^{\mathrm{a}}$

a Derived from eq. (2) using median $M / L$ values for groups with $N_{g} \geq 3$. 
measured in the dark-matter models, and integrated $M / L$ ratios deduced. Virial mass estimates were made and the inferred $M / L$ values compared to the global mean value and, in the dark-matter models, to the local mass-to-light ratio measured within the volume of the group occupied by the galaxies.

The main results can be summarized as follows.

1. In the simulations, the frequency of small groups $\left(N_{g} \leq\right.$ 4 ), which contain $\sim 65 \%$ of all galaxies, is very similar to that of the observational data. Group sizes and velocity dispersions, however, are not simultaneously reproduced within any single model.

2. Segregation of galaxies from dark matter occurs during the evolution of the two-component groups. The integrated mass-to-light profile, $M / L(<r)$, defined as the ratio of total to luminous mass within a radius $r$ of the group centroid, rises as roughly $r^{0.5}$ from values of $\sim 2$ in the inner regions to a value $M / L(<r) \approx 6$, thereafter rising more slowly the gobal mean value $M / L_{\text {glob }}=10$. This mean behavior is independent of the number of galaxies in the group.

3. The local mass-to-light ratio measured within the volume of the group occupied by galaxies is $\sim 35 \%$ of the global value, indicating that nearly two-thirds of the total mass associated with groups lies outside their visible regions.

4. The median virial mass estimates in runs with no dark matter are accurate to within $\sim 20 \%$ for the ensemble of groups with three or more members.

5. In the dark-matter models, the median virial $M / L$ 's for groups with three or more members underestimate the global $M / L$ by factors of $5(\Omega=1)$ and $8\left(\Omega_{f}=0.15\right)$. Using the properly softened form of the particle acceleration reduces the discrepancies to factors of $\sim 3$ and 4 , respectively.
6. The softened virial $M / L$ 's do accurately measure the local mass-to-light ratio within the volume of the group occupied by galaxies.

The relationship between the luminous and total mass profiles in the dark halo simulations is qualitatively similar to the relationship between luminosity and binding mass densities for the poor X-ray clusters studied by Kriss et al. (1983). This may be taken as an indication that the dynamics in the models is producing an accurate reflection of the segregation occurring between dark and luminous mass in real clusters. The models would then predict that substantial amounts of mass lie beyond the luminous confines of groups and clusters, in a fashion analogous to the dark halos which surround individual galaxies. Estimates of $\Omega_{0}$ based on group $M / L$ ratios would then underestimate the true value considerably. The value $\Omega_{0} \approx 0.2$ inferred by existing observations could thus be reconciled with a true value of $\Omega_{0}=1$. This conclusion arises from models with no built-in mass segregation on scales encompassing a few galaxies or larger. The segregation is purely dynamical in origin, occurring as the galaxies in the two-component groups experience relaxation and dynamical friction during nonlinear clustering.

This work constitutes part of a doctoral thesis at SUNYStony Brook. The models were conceived at the Institute of Astronomy, Cambridge. I am grateful to the Institute for its hospitality and particularly to G. Efsthathiou for use of the $P^{3} M$ code. It is a pleasure to thank E. Baron, B. Paczyński, L. Spitzer, and A. Yahil for helpful comments on earlier versions of this paper. This work was funded by USDDE grant DEAC02-80ER10719 at the State University of New York.
Aarseth, S. J., Gott, J. R., and Turner, E. L. 1979, Ap. J., 228, 664

Albert, C. E., White, R. A., and Morgan, W. W. 1977, Ap.J., 211, 309.

Bahcall, J. N., and Casertano, S. 1985, Ap. J. (Letters), 293, L7.

Barnes, J. 1984, M.N.R.A.S., 208, 885.

Bothun, G. D., Geller, M. J., Beers, T. C., and Huchra, J. P. 1983, Ap. J., 268 47.

Davis, M., Efstathiou, G., Frenk, C. S., and White, S. D. M. 1985, Ap. J., 292, 371.

Dekel, A., and Rees, M. J. 1986, Nature, submitted (preprint).

Dressler, A. 1984, Ann. Rev. Astr. Ap., 22, 185.

Efstathiou, G., and Barnes, J. 1983, in Formation annd Evolution of Galaxies and Large Structures in the Universe, ed. J. Audouze and J. Tran Tranh Van (Dordrecht: Reidel), p. 361.

Efstathiou, G., and Eastwood, J. W. 1981, M.N.R.A.S., 194, 503.

Efstathiou, G., Fall, S. M., and Hogan, C. 1979, M.N.R.A.S., 189, 203.

Evrard, A. E. 1986, Ap. J., 310, 1 (Paper I).

Evrard, A. E., and Yahil, A. 1985a, Ap. J., 296, 299

. 1985b, Ap. J., 296, 310.

Faber, S., and Gallagher, J. 1979, Ann. Rev. Astr. Ap., 17, 135.

\section{REFERENCES}

Felten, J. E. 1985, Comm. Ap., 11, 53.

Frenk, C. S., White, S. D. M., Efstathiou, G., and Davis, M. 1985, Nature, 317, 595.

Geller, M. J. 1984, in Clusters and Groups of Galaxies, ed. F. Mardirossian et al. (Boston: Reidel), p. 353.

Geller, M. J., and Peebles, P. J. E. 1973, Ap. J., 184, 329

Gott, J. R., and Turner, E. L. 1976, $A p . J ., 209,1$.

Heisler, J., Tremaine, S., and Bahcall, J. N. 1985, Ap. J., 298, 8.

Huchra, J. P., and Geller, M. J. 1982, Ap. J., 257, 423 (HG).

Kaiser, N., and Davis, M. 1985, Ap. J., 297, 365.

Kriss, G. A., Cioffi, D. F., and Canizares, C. R. 1983, Ap. J., 272, 439.

Morgan, W. W., Kayser, S., and White, R. A. 1975, Ap. J., 199, 545.

Rees, M. J., and Ostriker, J. P. 1977, Ap. J., 179, 541.

Rood, H. J., and Dickel, J. R. 1978, Ap. J., 224, 724.

Smith, S. 1936, Ap. J., 83, 23.

Turner, E. L., Aarseth, S. J., Gott, J. R., Blanchard, N. T., and Mathieu, R. D. 1979, Ap.J., 228, 684 .

Turner, E. L., and Gott, J. R. 1976, Ap. J., 209, 6.

Zwicky, J. 1933, Helvetica Phys. Acta, 6, 110.

August E. EvRARD: Institute of Astronomy, Madingley Road, Cambridge CB3 0HA, England, UK 